\title{
ARF-derived peptide sensitizes ovarian cancer cells to Cisplatin
}

\author{
Jinhua Zhou ${ }^{1,2,3,4 \#}$, Yunfei Wang ${ }^{2,3,4,5}$, Yun Qian ${ }^{6}$, Xinran Wang ${ }^{2}$, Ting Liu ${ }^{2,3}$, Wen Di $^{2,3,4}$ \\ ${ }^{1}$ Department of Obstetrics and Gynecology, The First Affiliated Hospital of Soochow University, Suzhou 215006, China; ${ }^{2}$ Department of Obstetrics \\ and Gynecology, Renji Hospital, School of Medicine, Shanghai Jiao Tong University, Shanghai 200127, China; ${ }^{3}$ Shanghai Key Laboratory of \\ Gynecologic Oncology, Shanghai 200127, China; ${ }^{4}$ Shanghai Health Bureau Key Disciplines and Specialties Foundation, Shanghai 200127, China; \\ ${ }^{5}$ Department of Gynecology, Affiliated Hospital of Jining Medical University, Jining 272000, China; ${ }^{6}$ Department of Gynecology, Shanghai \\ Municipal Hospital of Traditional Chinese Medicine, Shanghai University of Traditional Chinese Medicine, Shanghai 200071, China \\ Contributions: (I) Conception and design: J Zhou, W Di; (II) Administrative support: W Di; (III) Provision of study materials: Y Wang, T Liu; \\ (IV) Collection and assembly of data: J Zhou, Y Wang; (V) Data analysis and interpretation: J Zhou, Y Qian, X Wang; (VI) Manuscript writing: All \\ authors; (VII) Final approval of manuscript: All authors. \\ \#These authors contributed equally to this work. \\ Correspondence to: Wen Di. Department of Obstetrics and Gynecology, Renji Hospital, School of Medicine, Shanghai Jiao Tong University, 1630 \\ Dong Fang Road, Shanghai 200127, China. Email: diwen163@163.com; research123hop@163.com.
}

Background: Forkhead box M1 (FOXM1) is over-expressed in multiple cancers, and its over-expression induces chemoresistance and contributes to unfavorable prognosis in patients with ovarian cancer. P19 ${ }^{\mathrm{ARF}}$ is a tumor suppressor that contains a motif, which suppresses the transcriptional activity of FOXM1. In this study we investigated the potential role of alternative reading frame (ARF)-derived peptide as a cisplatin sensitizer for ovarian cancer.

Methods: FOXM1 expression in ovarian cancer tissues were analyzed using the online databases Oncomine and protein atlas. The association between FOXM1 expression and cisplatin response was analyzed and plotted in a panel of ovarian cancer cell lines. The expression of FOXM1 downstream target genes were accessed in SKOV3 cells treated with ARF peptide using quantitative polymerase chain reaction (QPCR) and western blot. Clonogenicity and apoptosis in SKOV3 cells treated with ARF peptide in the presence or absence of cisplatin was used to evaluate the effect of ARF peptides. In addition, tumor size in nude mice bearing SKOV3 xenografts were measured in the presence or absence of wild-type or mutant ARF peptide and with or without cisplatin.

Results: FOXM1 was over-expressed in ovarian cancer tissues and cell lines. FOXM1 expression was associated with cisplatin response in the ovarian cancer cell lines. ARF peptide decreased both mRNA and protein expression of FOXM1 downstream target genes, polo-like kinase 1 (PLK1) and exonuclease 1 (EXO1). In vitro, SKOV3 cells pretreated with ARF peptide showed increased apoptotic cells, decreased clonogenicity, and increased cleavage of caspase-3 in response to cisplatin treatment. In vivo, combination of wild-type ARF peptide and cisplatin had increased inhibition of tumor growth compared to mutant peptide.

Conclusions: FOXM1 expression levels are associated with cisplatin response. Treatment with ARF peptide enhanced cisplatin sensitivity in ovarian cancer cells. These results suggest a new therapeutic strategy to overcome chemoresistance in FOXM1 overexpressing ovarian cancers.

Keywords: Forkhead box M1 (FOXM1); ovarian cancer; alternative reading frame (ARF); cisplatin; peptide

Submitted Apr 06, 2018. Accepted for publication Nov 28, 2018.

doi: $10.21037 /$ tcr.2018.12.06

View this article at: http://dx.doi.org/10.21037/tcr.2018.12.06 


\section{Introduction}

Ovarian cancer remains one of the most lethal malignancies worldwide that targets the female reproductive system (1). Although overall survival and progression-free survival have increased after cisplatin-based chemotherapy, nearly $30 \%$ of ovarian cancers respond poorly, leading to resistant and recurrent disease (2). Hence, there is a clear unmet medical need to develop novel therapies to target chemo-resistance in patients with ovarian cancer.

FOXM1 belongs to the Forkhead box (Fox) transcription factor family and is an important regulator of the cell cycle (3). This proliferation-specific transcription factor is over-expressed in the majority of human cancers, including lung, gastrointestinal, mammary, glioblastoma, and ovarian cancer (4). Previous studies have shown that the FOXM1 transcription factor and its direct transcriptional targets were significantly altered in $87 \%$ of high-grade ovarian cancers (5). FOXM1 promotes cell proliferation, migration and invasion (6) and plays a significant role in chemoresistance in several solid cancers, including ovarian cancer (7-10). Hence, FOXM1 could be used as an effective drug discovery platform to identify chemo-sensitizing agents (11).

The $A R F / I N K 4 A$ locus encodes two distinct tumor suppressors, the $\mathrm{p} 16^{\mathrm{INK} 4 \mathrm{~A}}$ and the $19 \mathrm{kDa}$ alternative reading frame (ARF) protein (14 $\mathrm{kDa}$ in human) $(12,13)$. The synthesized (D-Arg)9-p19 ${ }^{\mathrm{ARF}}$ peptide that consists of amino acids 26-44 of $\mathrm{p} 19^{\mathrm{ARF}}$ protein has been demonstrated to inhibit hepatocellular cancer and osteosarcoma by decreasing FOXM1 transcriptional activity $(12,14,15)$. Furthermore, the ARF peptide inhibitor of FOXM1 sensitized breast cancer cells to Herceptin and paclitaxel treatment (7).

Our previous study demonstrated that FOXM1 contributes to cisplatin response in ovarian cancer cell lines (10). Here, we further investigated the effect of ARF peptide on cisplatin sensitization in SKOV3 ovarian cancer cells with aberrant FOXM1 expression. We found that ARF peptide sensitizes SKOV3 cells to cisplatin in vitro and in vivo.

\section{Methods}

\section{Cell culture, chemotherapeutic agents and animal models}

The human serous ovarian cancer cell lines A2780, SKOV3, ES2, HO8910 (16), and 3AO (17) were purchased from the Cell Bank of the Chinese Academy of Science (Shanghai, China). SKOV3 cells were cultured in Mccoy's 5A (Hyclone, USA), while all other cell lines were cultured in RPMI
1640 (Hyclone, USA). Media was supplemented with $10 \%(\mathrm{v} / \mathrm{v})$ fetal bovine serum, 100 units $/ \mathrm{mL}$ penicillin and $100 \mathrm{\mu g} / \mathrm{mL}$ streptomycin. Cells were maintained at $37^{\circ} \mathrm{C}$ in a humidified atmosphere containing $5 \% \mathrm{CO}_{2} / 95 \%$ air. Cisplatin was purchased from Sigma-Aldrich (Louis, MO, USA). Female nu/nu mice (5-6 weeks old, body weight 18-20 g) were purchased from Shanghai Slac Laboratory Animal Co., Ltd. (Shanghai, China) and housed under SPF conditions. Animal procedures were performed in accordance to approved protocols established by the Animal Care and Use Committee of Shanghai Jiao Tong University, School of Medicine.

\section{Western blot analysis}

Cells were lysed in radioimmunoprecipitation assay (RIPA) buffer supplemented with phenylmethylsulfonyl fluoride (PMSF). Primary human ovarian surface epithelium (HOSE) cell lysates were purchased from Pricell (Wuhan, China). Protein concentrations were determined using the Pierce ${ }^{\mathrm{TM}}$ BCA Protein Assay Kit (Grand Island, NY, USA). Equal amounts of cell lysates $(10-20 \mu \mathrm{g})$ were resolved by SDS-PAGE and electro-transferred to polyvinylidene difluoride (PVDF) membrane (Millipore). The following antibodies were used for western blotting: human FOXM1 (1:100, Santa Cruz Biotechnology), cleaved caspase-3 (1:1,000, Cell Signaling Technology), PLK1 (1:1,000, Abcam), EXO1 (1:100, Thermo Fisher Scientific), $\beta$-actin (1:2,000, Abcam).

\section{Cell viability assay}

Cell viability was measured using the Cell Counting Kit-8 assay (Dojindo Molecular Technologies) as previously described (10). Cells were plated at a density of $(2-5) \times 10^{3}$ cells/well on 96-well plates and subjected to different treatment conditions. Following 24-hour incubation at $37^{\circ} \mathrm{C}$ in a humidified incubator at $5 \% \mathrm{CO}_{2} / 95 \%$ air, the cells were subsequently incubated for an additional hour with CCK8 reagent. Cell viability was determined by measuring the absorbance at $450 \mathrm{~nm}$ using the $\mathrm{FL} \times 800$ Fluorescence Microplate Reader (Biotek).

\section{Flow cytometry}

Apoptosis was measured using flow cytometric analysis of Annexin $\mathrm{V}$ and propidium iodide (PI) staining (BD Bioscience, San Jose, CA, USA) according to the manufacturer's protocol. Cells were seeded into 6-well 
culture plates and incubated for 24 hours, then subjected to the indicated treatments. After treatment, cells were rinsed and resuspended in $100 \mu \mathrm{L}$ binding buffer and incubated at room temperature for $15 \mathrm{~min}$ with $5 \mu \mathrm{L}$ annexin V-FITC and $5 \mu \mathrm{L}$ of PI. Cells were then analyzed using the flow cytometer FC500/FC500-MPL (Beckman Coulter).

\section{Clonogenic assays}

Tumor cells were subjected to different treatments for the indicated times and then plated in 6-well culture plates at the density of 500 cells/well. Following incubation at $37^{\circ} \mathrm{C}$ in a humidified incubator at $5 \% \mathrm{CO}_{2} / 95 \%$ air for $8-10$ days, the media was changed every 3 days. At the end of the culture period, cells were stained with $1 \%$ methylene blue in $50 \%$ methanol for 20 minutes, washed with water. Cell colonies were counted using the ImageJ software at the same settings for all treatment conditions.

\section{Immunobistochemistry}

Immunohistochemistry was performed to assess antitumor efficacy in vivo as described previously (18). Ki-67 (1:100, Abcam) and cleaved caspase-3 (1:100, CST) antibody was used to assess proliferation and apoptosis.

\section{Synthesis of (D-Arg) ${ }_{9}-p 19^{A R F} 26-44$ peptide}

Apeptide company (Shanghai, China) synthesized wild-type $\mathrm{ARF}_{26-44}$ peptide (rrrrrrrKFVRSRRPRTASCALAFVN) and mutant $\mathrm{ARF}_{37-44}$ peptide (rrrrrrrrrSCALAFVN) as previously described (14). Both the peptides contained nine D-Arg ( $r$ ) residues at the $\mathrm{N}$ terminus, which had been demonstrated to promote cellular uptake of polypeptides (19). The peptides were tagged with fluorescent Lissamine (TRITC) on the $\mathrm{N}$ terminus and acetylated at the $\mathrm{C}$ terminus.

\section{Promoter reporter and luciferase assays}

Mutant and wild-type pGL3-FHRE2 reporter constructs were generated as described previously (10). Luciferase activity was measured following the luciferase assay system protocol (Promega).

\section{Murine xenograft model}

Female BALB/c nude mice (6-8 weeks old; body weight
18-20 g) were purchased from Shanghai Laboratory Animal Co., (SLAC, Shanghai, China) and housed under specific pathogen-free conditions. Tumor xenografts were established by subcutaneous inoculation of a suspension of SKVO3 cells $\left(2 \times 10^{6} / 100 \mu \mathrm{L}\right)$. Animals were monitored until tumor volume reached approximately $100 \mathrm{~mm}^{3}$. The Animal Care and Use Committee of Renji Hospital, Shanghai Jiao Tong University, School of Medicine (No. XS2016-005) approved the experimental protocols. All animal experiments were carried out in accordance with the UK Animals (Scientific Procedures) Act of 1986 and associated guidelines. Animal studies adhered to the ARRIVE guidelines.

\section{Therapeutic efficiency}

When the SKOV3 xenograft tumor volume reached $100 \mathrm{~mm}^{3}$, cancer-bearing mice were randomly divided into three groups. The three groups were then separately treated with intraperitoneal injection of cisplatin $(5 \mathrm{mg} / \mathrm{kg}$ body weight every 3 days over a 21-day period) in combination with intra-tumoral injection with wild-type or mutant ARF peptide $(5 \mathrm{mg} / \mathrm{kg}$ body weight, in $25 \mu \mathrm{L} \mathrm{PBS}, \mathrm{pH} 7.4$, every 3 days over a 21-day period), or with intraperitoneal injection with saline (non-treatment control). The therapeutic efficacy was determined by measuring tumor size of the xenografts. The size of the tumors were measured using calipers once every 3 days. The tumor volume was calculated according to the following formula: $\mathrm{V}=$ (largest diameter $\times$ small diameter $\left.{ }^{2}\right) \times \pi / 6$. Systemic toxicity was evaluated by monitoring changes to body weight.

\section{Statistical analysis}

SPSS18.0 software was used for statistical analysis. Asterisks in each graph denotes statistically significant changes, with $\mathrm{P}$ values calculated using Student $t$-test*, $\mathrm{P}<0.05$. $\mathrm{P}$ values of $<0.05$ were considered statistically significant.

\section{Results}

\section{FOXM1 is bighly expressed in ovarian cancer tissue.}

Using three independent ovarian cancer datasets: GSE12470 (Yoshihara Ovarian) (20), Welsh Ovarian (21), and GSE26712 (Bonome Ovarian) (22) in Oncomine (http:// www.oncomine.org), the $\log _{2}$ expression levels of FOXM1 in normal ovary and ovarian cancer tissues were plotted 


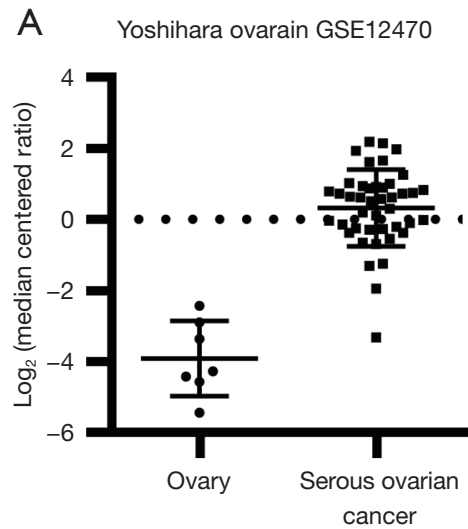

D

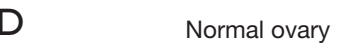

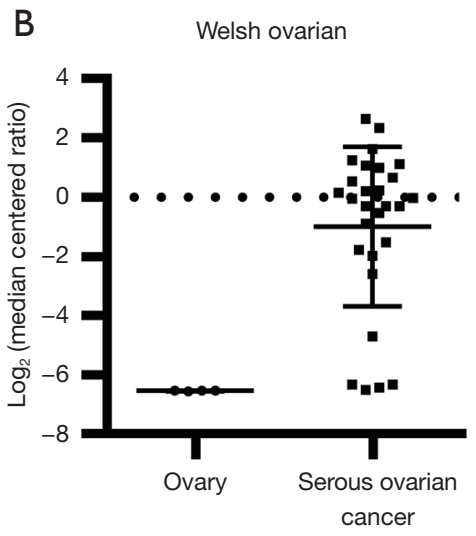

C

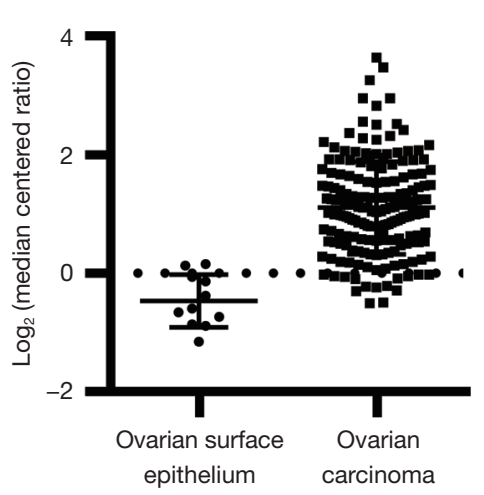

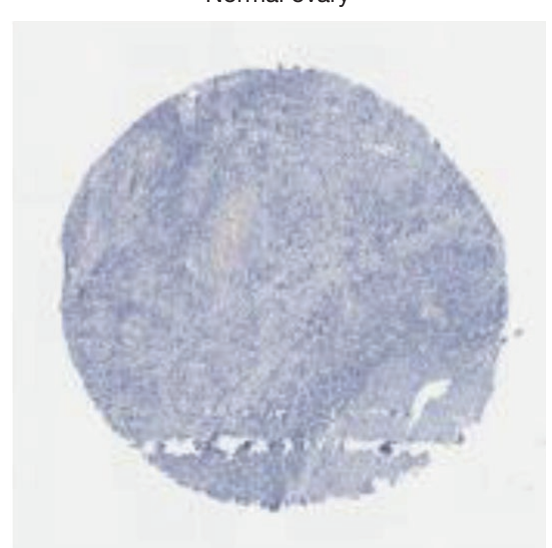

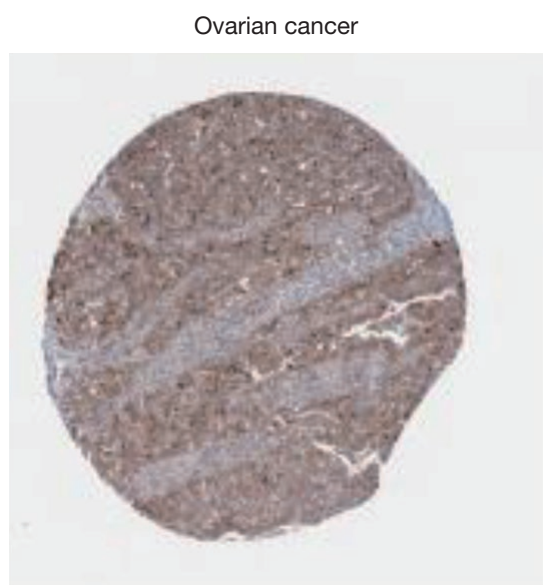

Figure 1 FOXM1 is highly expressed in ovarian cancer. (A) The $\log _{2}$ expression of probe A_23_P151150 representing FOXM1 in GSE12470 (Yoshihara Ovarian); (B) the $\log _{2}$ expression of probe U74612_at representing FOXM1 in Welsh Ovarian; (C) the log 2 expression of probe 202580_x_at representing FOXM1 in GSE26712; (D) representative images of FOXM1 expression in normal ovary and ovarian cancer by immunohistochemistry staining.

based on the probe representing FOXM1 mRNA. As shown in Figure 1A,B,C, FOXM1 was consistently over-expressed in ovarian cancer tissue compared to normal ovary or normal ovary epithelium, with fold change of 18.939 in GSE12470, 46.736 in Welsh Ovarian and 3.1 in GSE26712. In addition, FOXM1 protein expression was analyzed in ovarian cancer and normal ovary tissue using The Human Protein Atlas Database (http://www.proteinatlas.org/). Immunohistochemistry staining using three primary antibodies showed that FOXM1 was expressed at medium to high levels in 10 of 12 samples, however, FOXM1 expression was not detected in 3 samples of normal ovary tissue (Figure 1D). These data suggest that FOXM1 was aberrantly expressed in ovarian cancer.

\section{FOXM1 expression is associated with cisplatin response in ovarian cancer}

Previous studies have shown that FOXM1 regulates chemo-sensitivity to DNA damaging reagents in several cancers (11). To determine the relationship between FOXM1 expression and cisplatin response in ovarian cancer, we evaluated FOXM1 expression in five serous ovarian cancer cell lines [A2780, SKOV3, ES2, HO8910 (16), 3AO (17)]. Modest to robust expression of FOXM1 protein was detected in all cancerous cell lines but not in primary ovarian epithelium cells (OSE) (Figure 2A). The $\mathrm{IC}_{50}$ of cisplatin arranged from 12.7 to $30.1 \mu \mathrm{g} / \mathrm{mL}$ with 12.7 in ES2 cells, 17.3 in A2780 cells, 19.8 in HO8910 cells, 23.5 in $3 \mathrm{AO}$ cells and 30.1 in SKOV3 cell (Figure 2B). 
A

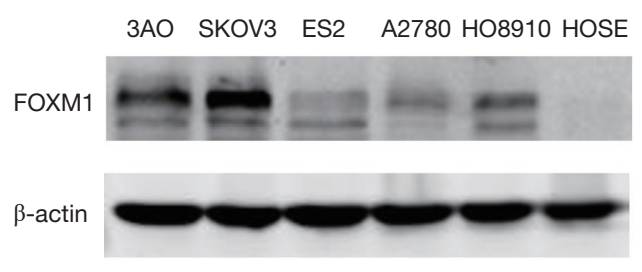

B

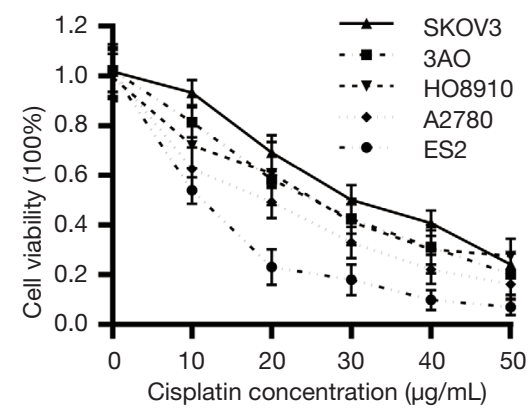

C

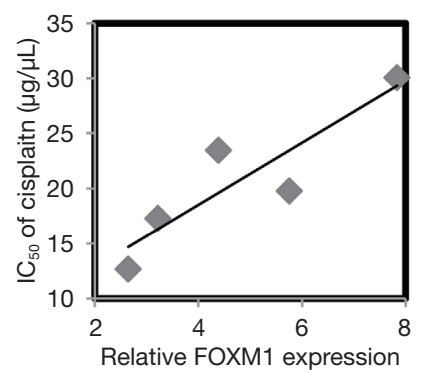

Figure 2 FOXM1 expression is inversely correlated with cisplatin sensitivity. (A) Lysates from indicated ovarian cancer cell lines and HOSE cells were subjected to western blot analysis for FOXM1 expression; (B) indicated cells were plated at density of 3,000-8,000/well in 96-well plate, then treated with cisplatin for 24 hours. Cell viability was detected by CCK 8 assay; (C) dot plot showing the correlation between FOXM1 expression and $\mathrm{IC}_{50}$ for each cell line examined. HOSE, human ovarian surface epithelium.

More importantly, FOXM1 expression in the cell lines was positively correlated with the $\mathrm{IC}_{50}$ of cisplatin. The correlation index (Pearson correlation) was $0.857(\mathrm{P}<0.05)$ (Figure 2C). Together, these results indicated that FOXM1 expression was associated with cisplatin response in ovarian cancer cell lines.

\section{ARF peptide inhibits expression of FOXM1 downstream target genes in SKOV3 cell line}

Previous studies have demonstrated that membranetransducing (D-Arg) $)_{9}-\mathrm{p} 19^{\mathrm{ARF}}$ peptide inhibited FOXM1 transcriptional activity in human osteosarcoma U2OS cells (14), and induced apoptosis in human hepatoma HepG2 cells (15). We investigated whether ARF peptide had similar effect in ovarian cancer cells. First, ARF peptide with TRITC tag was synthesized and cell permeability of the peptides was evaluated in SKOV3 cells with high $\mathrm{IC}_{50}$ of cisplatin and high expression of FOXM1 (Figure 3A). As expected, the ARF peptide penetrated the cell membrane and translocated into the nucleus of SKOV3 cells after incubation for 12 hours (Figure 3B). When cell viability was measured in SKOV3 cells treated with wild-typed or mutant peptides, the wild-type peptide was found to inhibit SKOV3 cell growth with an $\mathrm{IC}_{50}$ of $25 \mu \mathrm{M}$, while the mutant peptide only showed minor inhibition for cell proliferation (Figure 3C). To confirm previous reports that FOXM1 could regulate the transcription of PLK1 (3), EXO1 (9) and whether the ARF-derived peptide could inhibit FOXM1 transcriptional activity (15), we measured PLK1 and EXO1 gene expression in SKOV3 cells. QPCR and Western blot analysis demonstrated that PLK1 and EXO1 expression was downregulated at both the mRNA and protein level after treatment with $5 \mu \mathrm{M}$ wild-type ARF peptide but had a minimum effect on cell viability (Figure 3D,E). This result suggested that ARF peptide could directly target the transcription of PLK1 and EXO1 and that downregulation of gene expression was not due to fewer viable cells. In addition, reporter assays demonstrated that the ARF peptide affected luciferase activity in wild-type pGL3-FHRE2 constructs having the EXO1 promoter, but not in the mutant FHRE2 construct (Figure 3F). The results demonstrate that the ARF peptide could suppress FOXM1 transcriptional activity in SKOV3 cells.

\section{ARF peptide sensitizes ovarian cancer to cisplatin in vitro}

Since FOXM1 expression levels are associated with cisplatin response in ovarian cancer, and ARF peptide could inhibit FOXM1 transcriptional activity in SKOV3 cells, we hypothesized that the ARF peptide could sensitize ovarian cancer cells to cisplatin in vitro. To test this, SKOV3 cells were incubated with $5 \mu \mathrm{M}$ ARF peptide for 24 hours, then treated with cisplatin for an additional 24 hours. Then cell viability was normalized to the $\mathrm{OD}_{450}$ value of cells treated with peptide alone to eliminate any growth inhibitory effect of the peptide. The results showed that cells pretreated with wild-type peptide were more sensitive to cisplatin compared to cells pretreated with mutant peptide ( $22.4 v s . \sim 31.7 \mu \mathrm{g} / \mathrm{mL}$ ) (Figure $4 A$ ). In addition, colony formation was evaluated in SKOV3 cells treated with peptides in the presence or absence of 

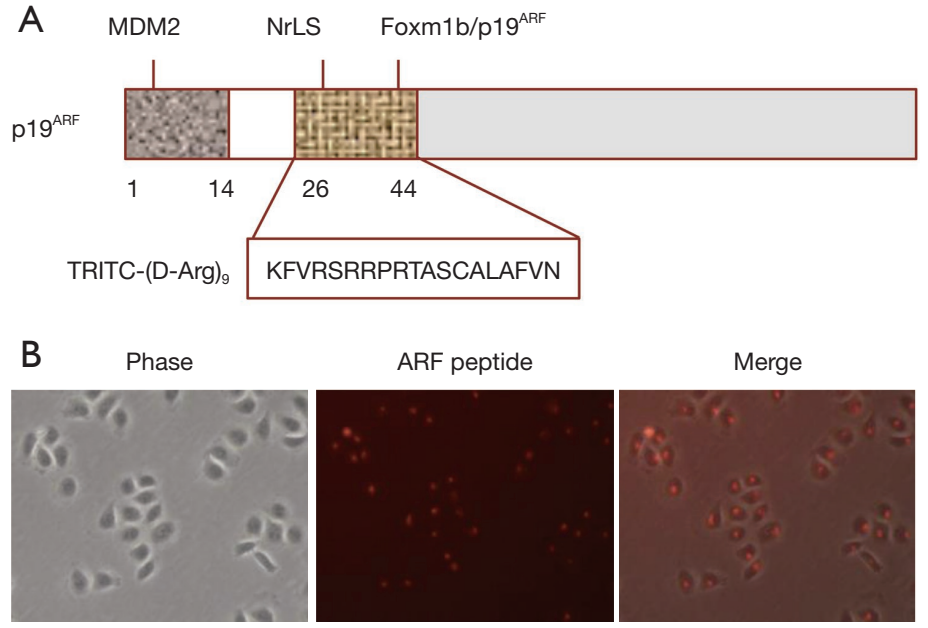

D

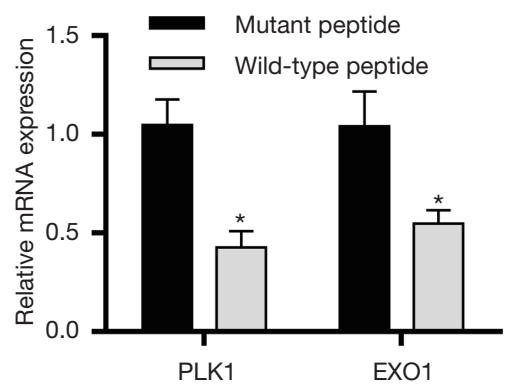

$E$

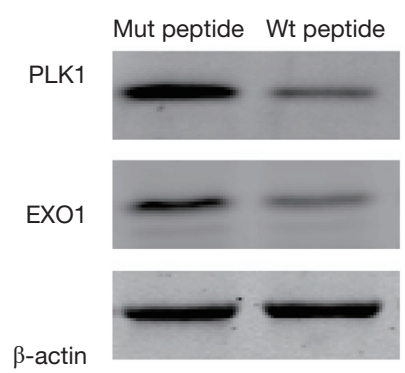

C

SKOV3

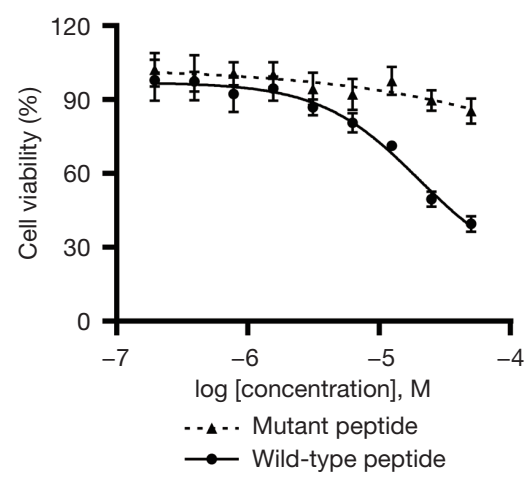

$\mathrm{F}$

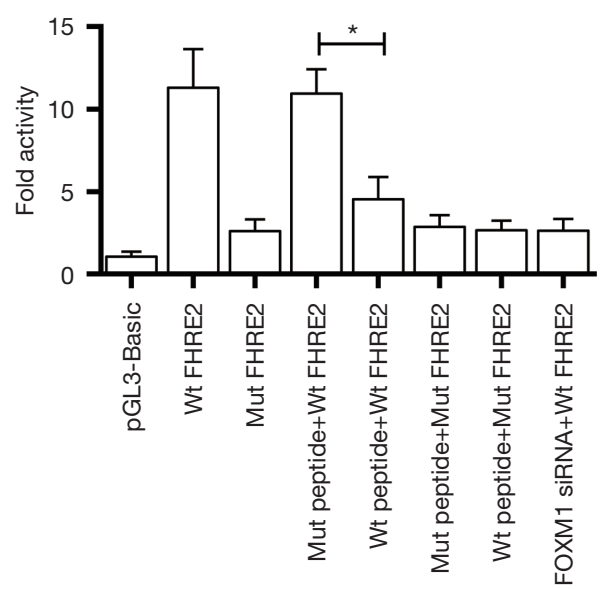

Figure 3 ARF peptide inhibits expression of FOXM1 downstream target genes in SKOV3 cells. (A) The schematic structure of p19 ${ }^{\mathrm{ARF}}$ protein and the sequence of the ARF peptide; (B) SKOV3 cells were plated in 6-well plates and treated with TRITC-peptide for 12 hours. Images were captured with bright light in phase contrast or with excitation at $532 \mathrm{~nm}$ laser; (C) SKOV3 cells were plated in 6-well plates and treated with increasing concentrations of wild-type or mutant TRITC-peptide for 48 hours. The response curve for the indicated peptide in SKOV3 cells are shown; (D) SKOV3 cells were treated the same as in (C), then mRNA expression of PLK1 and EXO1 were analyzed by QPCR; (E) SKOV3 cells were treated the same as in (C), then cell lysates were subjected to western blot analysis for PLK1 and EXO1 protein expression; (F) SKOV3 cells were transfected with the indicated construct derived from EXO1 promoter and/or siRNA, then treated with wild-type or mutant TRITC-peptide for 24 hours. Luciferase reporter assays were then performed and luciferase activity was normalized to pGL3-Basic. The results from three independent experiments were plotted. * $\mathrm{P}<0.05$. ARF, alternative reading frame.

cisplatin. Wild-type peptide pretreated cells exhibited attenuated colony formation ability after cisplatin treatment $(19.6 \% \pm 6.40 \%$ vs. $40.4 \% \pm 9.1 \%)$, with peptide treatment alone showing minimal effects on colony formation (Figure $4 B, C$ ). Apoptosis analysis by flow cytometry also showed that apoptosis was significantly higher in cells pretreated with wild-type peptide compared to mutant peptide $(36.5 \% \pm 3.0 \%$ vs. $20.7 \% \pm 3.5 \%)$, however peptide treatment alone did not induced notable apoptosis (Figure $4 D, E$ ). Consistent with this, western blot analysis showed significant enhanced expression of cleaved caspase-3 in wild-type ARF pretreated cells (Figure 4F). These results indicate that ARF peptide could sensitize SKOV3 cells to cisplatin treatment, and increased cell apoptosis induced by cisplatin contributed, at least partially, to enhancing cytotoxicity in cells pretreated with ARF peptide. 
A

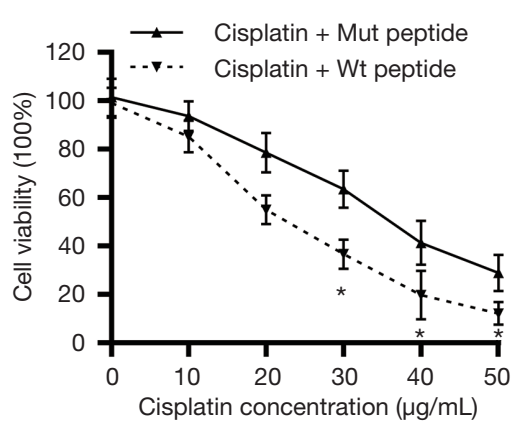

D

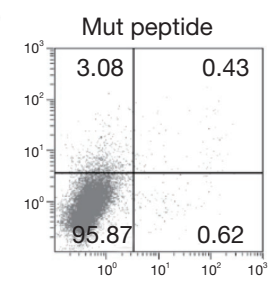

Cisplatin + Mut peptide
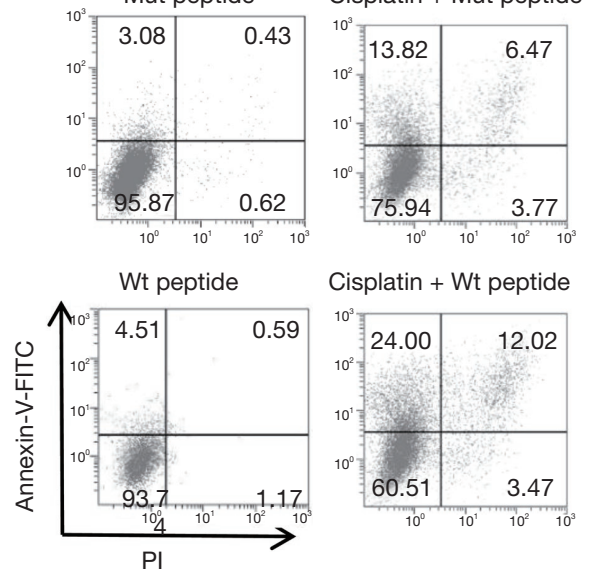

B

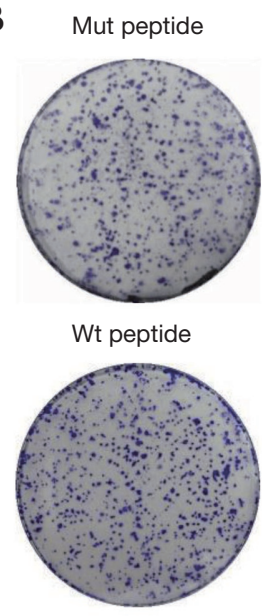

E

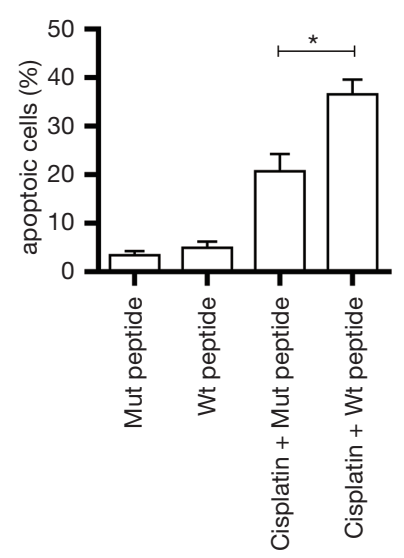

C

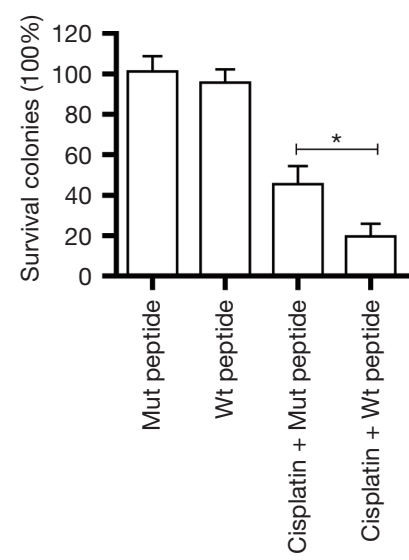

$\mathrm{F}$

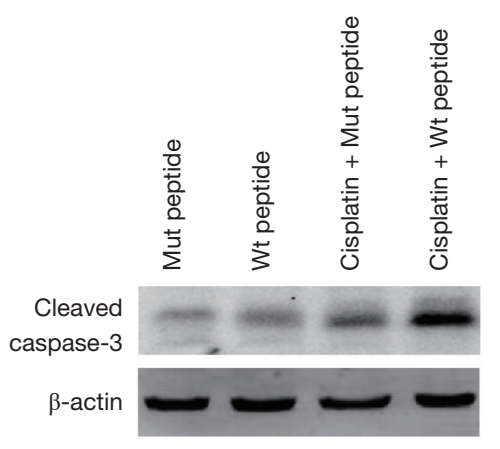

Figure 4 ARF peptide sensitizes SKOV3 cells to cisplatin in vitro. (A) SKOV3 cells were plated in 96-well plates at a density of 5,000/well, then incubated with wild-type ARF peptide or mutant peptide for 24 hours and with increasing concentration of cisplatin for an additional 24 hours. Cell viability was measured using the CCK8 assay; (B) twenty-four hours after $5 \mu \mathrm{M}$ of wild-type or mutant peptide treatment, SKVO3 cells were treated for 1 hour with $2 \mu \mathrm{g} / \mathrm{mL}$ cisplatin. Cells were then plated in 6-well plates. Colonies were stained and counted after incubation for 10 days. Results shown are representative of three independent experiments; (C) quantification as a percentage of colonies in cells treated with mutant peptide; (D) SKOV3 cells were plated in 6-well plates, and incubated with $5 \mu \mathrm{M}$ of wild-type ARF peptide or mutant peptide for 24 hours and with cisplatin $(20 \mu \mathrm{g} / \mathrm{mL})$ for an additional 24 hours. Apoptosis was then measured; (E) the percentage of apoptotic cells for each group; (F) SKOV3 cells were plated in 6-well plates, and incubated with $5 \mu M$ of wild-type or mutant peptide for 24 hours and with cisplatin $(10 \mu \mathrm{g} / \mathrm{mL})$ for an additional 24 hours. Cell lysates were collected for western blot analysis of cleaved caspase-3 expression. *, $\mathrm{P}<0.05$. ARF, alternative reading frame.

\section{ARF peptide sensitizes ovarian cancer to cisplatin in vivo}

In order to determine the effect of ARF peptide on tumor xenograft growth, mice bearing tumors were treated with saline, or with the combination of cisplatin and wide-type or mutant ARF peptide, and observed for 7 consecutive treatments (flow diagram shown in Figure $5 A$ ). As shown in Figure 5B, SKOV3 tumor growth was significantly inhibited in mice treated with cisplatin and mutant peptide, but, the combination of cisplatin with wild-type peptide produced significantly greater inhibition of tumor growth. There was no significant differences in body weight between mice treated with wild-type or mutant peptide (data not shown). To investigate the mechanism underlying the sensitization effect of ARF peptide observed in vitro and in vivo, we 
A

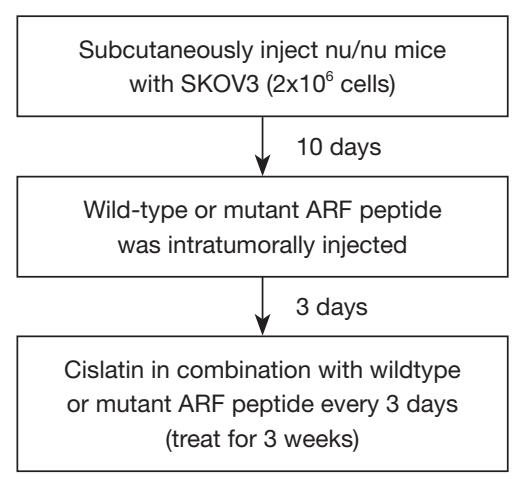

B

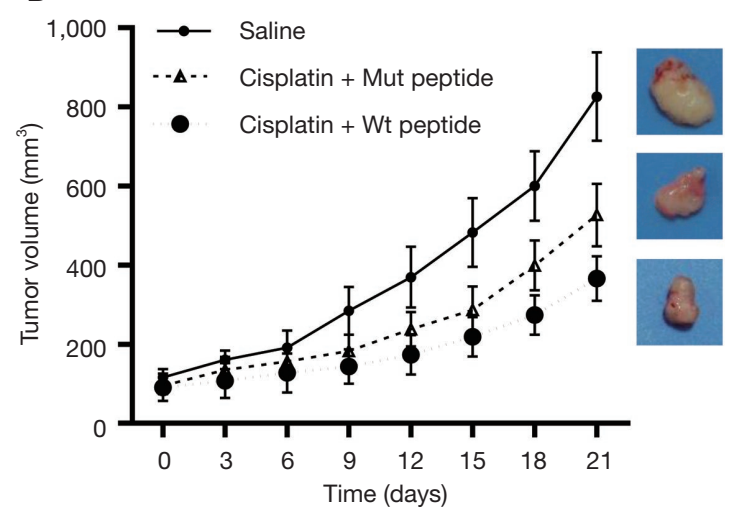

C

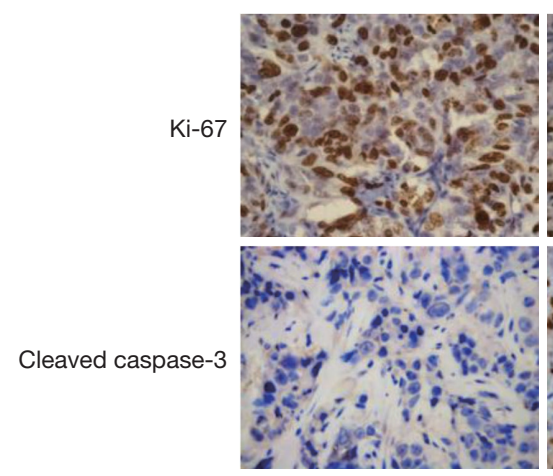

$\mathrm{D}$

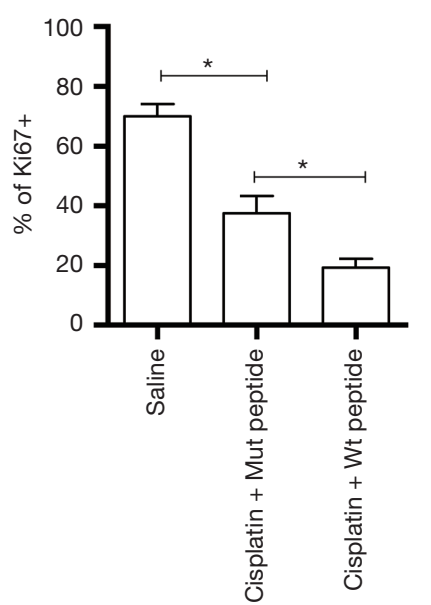

Cisplatin + Mut peptide

Cisplatin + Wt peptide
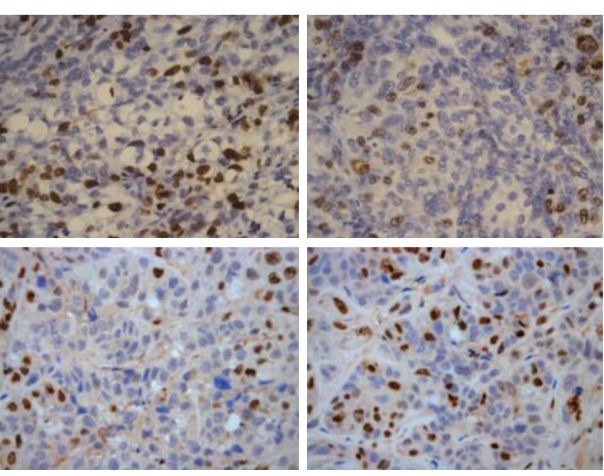

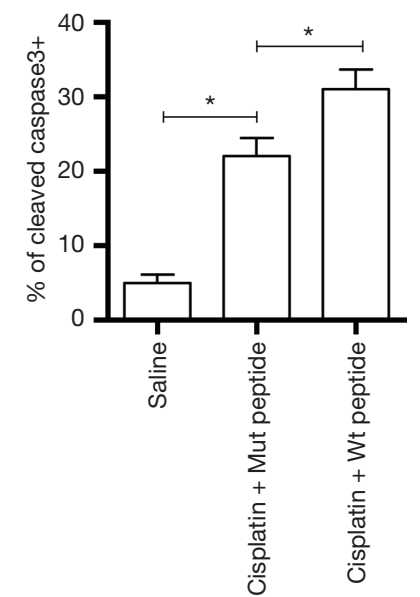

Figure $5 \mathrm{ARF}$ peptide sensitizes SKOV3 cells to cisplatin in vivo. (A) Flow diagram showing the design of the in vivo experiment; (B) tumor growth curve for each group based on measurements taken at 10, 13, 16, 19, 22, 26, 28 and 31 days after implantation; (C) immunohistochemical images of tumor tissues after the different treatments are presented for Ki67 and cleaved caspase-3 staining; (D) the percentage of Ki-67 or cleaved caspase3 positive cells for each group. * $\mathrm{P}<0.05$; ARF, alternative reading frame.

analyzed cancer proliferation and apoptosis in the tumors of treated mice (Figure 5C). Immunohistochemistry analysis demonstrated that the combination of cisplatin and wildtype ARF peptide produced a significant increase in cleaved caspase-3 $(\mathrm{P}<0.05)$ and significant decrease in Ki-67 $(\mathrm{P}<0.05)$ (Figure $5 D$ ), compared to the combination of cisplatin and mutant peptide.

\section{Discussion}

Patients with ovarian cancer have a poor prognosis because of late diagnosis and unfavorable response to chemotherapy. Targeted therapies are currently being investigated to find novel strategies to overcome chemoresistance. The most intensively studied molecular targets include
VEGF, EGFR, HER2, and PARP (23). Recent studies have shown that FOXM1, a key regulator of cell cycle progression, is aberrantly expressed in ovarian cancer and confers resistance to DNA damaging reagents including cisplatin (11). The $\mathrm{p} 19^{\mathrm{ARF}}$ residues 26-44 were sufficient to inhibit FOXM1 transcriptional activity and sensitize breast cancer cells to Herceptin and Taxol treatment $(7,15)$. We investigated whether cell-penetrating ARF peptide sensitizes ovarian cancer to cisplatin treatment. We showed that FOXM1 mRNA and protein were both highly expressed in ovarian cancer, and that FOXM1 expression levels inversely correlated with cisplatin response in ovarian cancer cell lines. SKOV3 cells treated with ARF peptide exhibited attenuated expression of FOXM1 downstream target genes, which may be due to the inhibition of FOXM1 
transcriptional activity. FOXM1 sensitized ovarian cancer to cisplatin in vitro and in vivo.

DNA-damaging drugs including platinum compounds are frequently used to treat solid tumors in the clinic. For ovarian cancer, cisplatin is the key constituent of postoperative primary chemotherapy as well as a neoadjuvant (24). The formation of cisplatin-DNA adducts leads to blockage of DNA, RNA, and protein synthesis resulting in apoptosis if the extent of damage is beyond repair. However, adverse effects such as, nephrotoxicity, neurotoxicity and ototoxicity limit the dosing of cisplatin (25). Multiple pathways and molecules contribute to cisplatin resistance (26). FOXM1, which is overexpressed in cancer, confers resistance to DNA damaging drugs and genotoxic agents by enhancing DNA repair gene networks (11). In addition, FOXM1 protects cancer cells from cisplatin-induced apoptosis (27). Small molecular inhibitors targeting FOXM1, such as Thiostrepton and siomycin A, have been proven as a potential strategy to overcome cisplatin resistance in cancer (28).

Peptide-based therapies have been developed for years with different anticancer mechanisms including tumor angiogenesis inhibition, tumor apoptosis or tumor necrosis induction, kinase activity inhibition. Several of these peptides have shown great promise in preclinical and clinical studies (29,30). In our study, ARF-derived peptide significantly inhibited the expression of FOXM1 downstream target genes, and sensitized ovarian cancer cells to cisplatin. These results suggest that FOXM1-targeting peptide is a novel therapeutic strategy to improve response to platinum-based chemotherapy in ovarian cancer patients.

\section{Conclusions}

Our study demonstrated that FOXM1 was aberrantly expressed and associated with cisplatin response in ovarian cancer. Cell-penetrating ARF-derived peptide inhibited expression of FOXM1 downstream target genes PLK1 and EXO1 and sensitized cancer cells to cisplatin in cultured SKOV3 cells and xenograft models. Our findings provide a new treatment strategy to improve cisplatin chemosensitivity in ovarian cancer.

\section{Acknowledgments}

We would like to thank all the lab members of Shanghai Key Laboratory of Gynecologic Oncology for supporting this study.
Funding: This study was supported by grants from the National Natural Science Foundation of China (No. 81272882, 81772773, 81302275, and 81672560), Jiangsu Provincial Medical Youth Talent Program (QNRC2016753), The Applied Basic Research Program of Science and Technology Commission Foundation of Suzhou (SYS201463), and The Project of Jiangsu Provincial Maternal and Child Health Association (No. FYX201709).

\section{Footnote}

Conflicts of Interest: All authors have completed the ICMJE uniform disclosure form (available at http://dx.doi. org/10.21037/tcr.2018.12.06). The authors have no conflicts of interest to declare.

Ethical Statement: The authors are accountable for all aspects of the work in ensuring that questions related to the accuracy or integrity of any part of the work are appropriately investigated and resolved. The study was conducted in accordance with the Declaration of Helsinki (as revised in 2013). Institutional ethical approval and informed consent were waived. The Animal Care and Use Committee of Renji Hospital, Shanghai Jiao Tong University, School of Medicine approved the animal experimental protocols (No. XS2016-005).

Open Access Statement: This is an Open Access article distributed in accordance with the Creative Commons Attribution-NonCommercial-NoDerivs 4.0 International License (CC BY-NC-ND 4.0), which permits the noncommercial replication and distribution of the article with the strict proviso that no changes or edits are made and the original work is properly cited (including links to both the formal publication through the relevant DOI and the license). See: https://creativecommons.org/licenses/by-nc-nd/4.0/.

\section{References}

1. Siegel RL, Miller KD, Jemal A. Cancer statistics, 2016. CA Cancer J Clin 2016;66:7-30.

2. Cannistra SA. Cancer of the ovary. $\mathrm{N}$ Engl J Med 2004;351:2519-29.

3. Laoukili J, Kooistra MR, Bras A, et al. FoxM1 is required for execution of the mitotic programme and chromosome stability. Nat Cell Biol 2005;7:126-36.

4. Gartel AL. FOXM1 in Cancer: Interactions and Vulnerabilities. Cancer Res 2017;77:3135-9. 
5. Cancer Genome Atlas Research Network. Integrated genomic analyses of ovarian carcinoma. Nature 2011;474:609-15.

6. Lok GT, Chan DW, Liu VW, et al. Aberrant activation of ERK/FOXM1 signaling cascade triggers the cell migration/invasion in ovarian cancer cells. PLoS One 2011;6:e23790.

7. Carr JR, Park HJ, Wang Z, et al. FoxM1 mediates resistance to herceptin and paclitaxel. Cancer Res 2010;70:5054-63.

8. Kwok JM, Peck B, Monteiro LJ, et al. FOXM1 confers acquired cisplatin resistance in breast cancer cells. Mol Cancer Res 2010;8:24-34.

9. Park YY, Jung SY, Jennings NB, et al. FOXM1 mediates Dox resistance in breast cancer by enhancing DNA repair. Carcinogenesis 2012;33:1843-53.

10. Zhou J, Wang Y, Wang Y, et al. FOXM1 Modulates Cisplatin Sensitivity by Regulating EXO1 in Ovarian Cancer. PLoS One 2014;9:e96989.

11. Zona S, Bella L, Burton MJ, et al. FOXM1: An emerging master regulator of DNA damage response and genotoxic agent resistance. Biochim Biophys Acta 2014;1839:1316-22.

12. Costa RH, Kalinichenko VV, Major ML, et al. New and unexpected: forkhead meets ARF. Curr Opin Genet Dev 2005;15:42-8.

13. Quelle DE, Zindy F, Ashmun RA, et al. Alternative reading frames of the INK4a tumor suppressor gene encode two unrelated proteins capable of inducing cell cycle arrest. Cell 1995;83:993-1000.

14. Kalinichenko VV, Major ML, Wang X, et al. Foxm1b transcription factor is essential for development of hepatocellular carcinomas and is negatively regulated by the p19ARF tumor suppressor. Genes Dev 2004;18:830-50.

15. Gusarova GA, Wang IC, Major ML, et al. A cellpenetrating ARF peptide inhibitor of FoxM1 in mouse hepatocellular carcinoma treatment. J Clin Invest 2007;117:99-111.

16. Yan HC, Fang LS, Xu J, et al. The identification of the biological characteristics of human ovarian cancer stem cells. Eur Rev Med Pharmacol Sci 2014;18:3497-503.

17. Shi MF, Jiao J, Lu WG, et al. Identification of cancer stem cell-like cells from human epithelial ovarian carcinoma cell line. Cell Mol Life Sci 2010;67:3915-25.

18. Wang Y, Liu P, Duan Y, et al. Specific cell targeting with APRPG conjugated PEG-PLGA nanoparticles for treating ovarian cancer. Biomaterials 2014;35:983-92.

19. Wender PA, Mitchell DJ, Pattabiraman K, et al. The design, synthesis, and evaluation of molecules that enable or enhance cellular uptake: peptoid molecular transporters. Proc Natl Acad Sci U S A 2000;97:13003-8.

20. Yoshihara K, Tajima A, Komata D, et al. Gene expression profiling of advanced-stage serous ovarian cancers distinguishes novel subclasses and implicates ZEB2 in tumor progression and prognosis. Cancer Sci 2009;100:1421-8.

21. Welsh JB, Zarrinkar PP, Sapinoso LM, et al. Analysis of gene expression profiles in normal and neoplastic ovarian tissue samples identifies candidate molecular markers of epithelial ovarian cancer. Proc Natl Acad Sci U S A 2001;98:1176-81.

22. Bonome T, Levine DA, Shih J, et al. A gene signature predicting for survival in suboptimally debulked patients with ovarian cancer. Cancer Res 2008;68:5478-86.

23. Tagawa T, Morgan R, Yen Y, et al. Ovarian cancer: opportunity for targeted therapy. J Oncol 2012;2012:682480.

24. Bookman MA. First-line chemotherapy in epithelial ovarian cancer. Clin Obstet Gynecol 2012;55:96-113.

25. Ruggiero A, Trombatore G, Triarico S, et al. Platinum compounds in children with cancer: toxicity and clinical management. Anticancer Drugs 2013;24:1007-19.

26. Galluzzi L, Senovilla L, Vitale I, et al. Molecular mechanisms of cisplatin resistance. Oncogene 2012;31:1869-83.

27. Halasi M, Gartel AL. Suppression of FOXM1 Sensitizes Human Cancer Cells to Cell Death Induced by DNADamage. PLoS One 2012;7:e31761.

28. Gartel AL. FoxM1 inhibitors as potential anticancer drugs. Expert Opin Ther Targets 2008;12:663-5.

29. Wu D, Gao Y, Qi Y, et al. Peptide-based cancer therapy: opportunity and challenge. Cancer Lett 2014;351:13-22.

30. Bidwell GL. Peptides for cancer therapy: a drugdevelopment opportunity and a drug-delivery challenge. Ther Deliv 2012;3:609-21.
Cite this article as: Zhou J, Wang Y, Qian Y, Wang X, Liu T, Di W. ARF-derived peptide sensitizes ovarian cancer cells to Cisplatin. Transl Cancer Res 2018;7(6):1614-1623. doi: 10.21037/tcr.2018.12.06 